\title{
Discrete Universality of Absolutely Convergent Dirichlet Series
}

\section{Mindaugas Jasas ${ }^{a}$, Antanas Laurinčikas ${ }^{a}$, Mindaugas Stoncelis ${ }^{b}$ and Darius Šiaučiūnas ${ }^{b}$}

${ }^{a}$ Institute of Mathematics, Faculty of Mathematics and Informatics, Vilnius University

Naugarduko g. 24, LT-03225 Vilnius, Lithuania

${ }^{b}$ Institute of Regional Development, Šiauliai Academy, Vilnius University

P. Višinskio g. 25, LT-76351 Šiauliai, Lithuania

E-mail: mindaugas.jasas@mif .stud.vu. It

E-mail: antanas.laurincikas@mif.vu.lt

E-mail: mindaugas.stoncelis@sa.vu.lt

E-mail(corresp.): darius.siauciunas@sa.vu.1t

Received June 7, 2021; revised November 17, 2021; accepted November 17, 2021

Abstract. In the paper, an universality theorem of discrete type on the approximation of analytic functions by shifts of a special absolutely convergent Dirichlet series is obtained. These series is close in a certain sense to the periodic zeta-function and depends on a parameter.

Keywords: limit theorem, periodic zeta-function, universality, weak convergence.

AMS Subject Classification: $11 \mathrm{M} 41$.

\section{Introduction}

Let $\mathfrak{a}=\left\{a_{m}: m \in \mathbb{N}\right\}$ be a periodic sequence of complex numbers with minimal period $q \in \mathbb{N}$. The periodic zeta-function $\zeta(s ; \mathfrak{a}), s=\sigma+i t$, is defined, for $\sigma>1$, by the Dirichlet series

$$
\zeta(s ; \mathfrak{a})=\sum_{m=1}^{\infty} \frac{a_{m}}{m^{s}} .
$$

Copyright (c) 2022 The Author(s). Published by Vilnius Gediminas Technical University This is an Open Access article distributed under the terms of the Creative Commons Attribution License (http://creativecommons.org/licenses/by/4.0/), which permits unrestricted use, distribution, and reproduction in any medium, provided the original author and source are credited. 
In view of periodicity of the sequence $\mathfrak{a}$,

$$
\zeta(s ; \mathfrak{a})=\frac{1}{q^{s}} \sum_{l=1}^{q} a_{l} \zeta\left(s, \frac{l}{q}\right),
$$

where $\zeta(s, \alpha), 0<\alpha \leqslant 1$, denotes the classical Hurwitz zeta-function, it follows that the function $\zeta(s ; \mathfrak{a})$ is analytic in the whole complex plane, except for a simple pole at the point $s=1$ with residue

$$
\hat{a} \stackrel{\text { def }}{=} \frac{1}{q} \sum_{l=1}^{q} a_{l} .
$$

If $a_{m} \equiv 1$, then the function $\zeta(s ; \mathfrak{a})$ reduces to the Riemann zeta-function $\zeta(s)$. If $a_{m}=\chi(m)$, where $\chi(m)$ is a Dirichlet character modulo $q$, then the function $\zeta(s ; \mathfrak{a})$ becomes a Dirichlet $L$-function $L(s, \chi)$. Thus, the periodic zeta-function is a generalization of the classical Riemann zeta-function $\zeta(s)$ and Dirichlet $L$ function $L(s, \chi)$.

As the functions $\zeta(s)$ and $L(s, \chi)$, the function $\zeta(s ; \mathfrak{a})$ for some sequences $\mathfrak{a}$ is universal in the sense that its shifts $\zeta(s+i \tau ; \mathfrak{a}), \tau \in \mathbb{R}$, approximate a wide class of analytic functions defined on the strip $D=\{s \in \mathbb{C}: 1 / 2<$ $\sigma<1\}$. Universality of the function $\zeta(s ; \mathfrak{a})$ was considered by various authors, among them B. Bagchi, J. Steuding, J. Kaczorowski, J. Sander, R. Macaitienè, R. Kačinskaitè, the second, third and fourth authors, and others. For a more precise account, we propose a survey paper [6]. The majority of the papers deal with the continuous universality of $\zeta(s ; \mathfrak{a})$ when $\tau$ in shifts $\zeta(s+i \tau ; \mathfrak{a})$ takes arbitrary real values. To our knowledge, the discrete universality of $\zeta(s ; \mathfrak{a})$, when $\tau$ in $\zeta(s+i \tau ; \mathfrak{a})$ takes real values from a certain discrete set, was discussed only in $[3,4,5]$ with multiplicative sequence $\mathfrak{a}$, i. e., $a_{m n}=a_{m} a_{n}$ for all $(m, n)=$ 1 and $a_{1}=1$. For example, Theorem 2 of [4] with $w(u) \equiv 1$ implies the following result. Denote by $\mathcal{K}$ the class of compact subsets of the strip $D$ with connected complements, and by $H_{0}(K)$ with $K \in \mathcal{K}$ the class of continuous non-vanishing functions on $K$ that are analytic in the interior of $K$. Let $\mathbb{P}$ be the set of all prime numbers, \#A denotes the cardinality of the set $A$, and, for $h>0$,

$$
L(\mathbb{P}, h, \pi)=\left\{(\log p: p \in \mathbb{P}), \frac{2 \pi}{h}\right\} .
$$

Under above notation, for every $K \in \mathcal{K}, f(s) \in H_{0}(K)$ and $\varepsilon>0$,

$$
\liminf _{N \rightarrow \infty} \frac{1}{N+1} \#\left\{0 \leqslant k \leqslant N: \sup _{s \in K}|\zeta(s+i k h ; \mathfrak{a})-f(s)|<\varepsilon\right\}>0
$$

provided that the sequence $\mathfrak{a}$ is multiplicative, and the set $L(\mathbb{P}, h, \pi)$ is linearly independent over the field of rational numbers $\mathbb{Q}$.

Latter, we will give a modified statement of the above theorem from [4], for it some notation is required.

In this paper, we will consider the discrete approximation of analytic functions by shifts of a certain absolutely convergent on $D$ Dirichlet series with coefficients depending on a parameter. These series is closely connected to the function $\zeta(s ; \mathfrak{a})$. 


\section{Definition of an absolutely convergent series}

As usual, denote by $\Gamma(s)$ the Euler gamma-function, $\theta>1 / 2$ is a fixed number, $u>0$ and

$$
l_{u}(s)=\frac{s}{\theta} \Gamma\left(\frac{s}{\theta}\right) u^{s}, \quad v_{u}(m)=\exp \left\{-\left(\frac{m}{u}\right)^{\theta}\right\}, \quad m \in \mathbb{N} .
$$

Define the series

$$
\zeta_{u}(s ; \mathfrak{a})=\sum_{m=1}^{\infty} \frac{a_{m} v_{n}(m)}{m^{s}} .
$$

Since the sequence $\mathfrak{a}$ is bounded, the latter series is absolutely convergent, say, for $\sigma>1 / 2$. In view of the Mellin formula

$$
\frac{1}{2 \pi i} \int_{a-i \infty}^{a+i \infty} \Gamma(s) b^{-s} \mathrm{~d} s=\mathrm{e}^{-b}, \quad a, b>0,
$$

we have the equality

$$
v_{u}(m)=\frac{1}{2 \pi i} \int_{\theta-i \infty}^{\theta+i \infty} \frac{1}{\theta} \Gamma\left(\frac{s}{\theta}\right)\left(\frac{m}{u}\right)^{-s} \mathrm{~d} s .
$$

The latter formula also implies the bound $v_{u}(m) \ll_{\theta, u} m^{-\theta}$. Moreover, $(2.1)$ shows that

$$
\begin{gathered}
\zeta_{u}(s ; \mathfrak{a})=\frac{1}{2 \pi i} \sum_{m=1}^{\infty} \frac{a_{m}}{m^{s}} \int_{\theta-i \infty}^{\theta+i \infty} \frac{z}{\theta} \Gamma\left(\frac{z}{\theta}\right)\left(\frac{m}{u}\right)^{-z} \frac{\mathrm{d} z}{z} \\
=\frac{1}{2 \pi i} \int_{\theta-i \infty}^{\theta+i \infty} \frac{l_{u}(z)}{z} \sum_{m=1}^{\infty} \frac{a_{m}}{m^{s+z}} \mathrm{~d} z=\frac{1}{2 \pi i} \int_{\theta-i \infty}^{\theta+i \infty} \zeta(s+z ; \mathfrak{a}) l_{u}(z) \frac{\mathrm{d} z}{z} .
\end{gathered}
$$

\section{Statement of the main theorem}

Denote by $H(D)$ the space of analytic functions on $D$ endowed with the topology of uniform convergence on compacta. In this section, we define the $H(D)$ valued random element connected to the function $\zeta(s ; \mathfrak{a})$. We start with traditional probability space. Let

$$
\Omega=\prod_{p \in \mathbb{P}} \gamma_{p}
$$

where $\gamma_{p}=\{s \in \mathbb{C}:|s|=1\}$ for all $p \in \mathbb{P}$. The torus $\Omega$ is a compact topological Abelian group, therefore, on $(\Omega, \mathcal{B}(\Omega))$, where $\mathcal{B}(\mathbb{X})$ is the Borel $\sigma$-field of the space $\mathbb{X}$, the probability Haar measure $m_{H}$ can be defined. This gives the probability space $\left(\Omega, \mathcal{B}(\Omega), m_{H}\right)$. Denote by $\omega(p)$ the $p$ th component of an element $\omega \in \Omega, p \in \mathbb{P}$. Now, on the probability space $\left(\Omega, \mathcal{B}(\Omega), m_{H}\right)$, define the $H(D)$-valued random element

$$
\zeta(s, \omega ; \mathfrak{a})=\prod_{p \in \mathbb{P}}\left(1+\sum_{\alpha=1}^{\infty} \frac{a_{p^{\alpha} \omega^{\alpha}(p)}}{p^{\alpha s}}\right) .
$$


The details of this definition can be found in [2]. We will prove the following statement.

Theorem 1. Suppose that the sequence $\mathfrak{a}$ is multiplicative, the set $L(\mathbb{P}, h, \pi)$ is linearly independent over $\mathbb{Q}$ and $u_{N} \rightarrow \infty, u_{N} \ll N^{2} \log ^{A} N$ with every $A>0$ as $N \rightarrow \infty$. Let $K \in \mathcal{K}$ and $f(s) \in H_{0}(K)$. Then the limit

$$
\begin{gathered}
\lim _{N \rightarrow \infty} \frac{1}{N+1} \#\left\{0 \leqslant k \leqslant N: \sup _{s \in K}\left|\zeta_{u_{N}}(s+i k h ; \mathfrak{a})-f(s)\right|<\varepsilon\right\} \\
=m_{H}\left\{\omega \in \Omega: \sup _{s \in K}|\zeta(s, \omega ; \mathfrak{a})-f(s)|<\varepsilon\right\}>0
\end{gathered}
$$

exists for all but at most countably many $\varepsilon>0$.

We also state a modified universality theorem for the function $\zeta(s ; \mathfrak{a})$ mentioned in Introduction.

Theorem 2. Suppose that the sequence $\mathfrak{a}$ is multiplicative, the set $L(\mathbb{P}, h, \pi)$ is linearly independent over $\mathbb{Q}$. Let $K \in \mathcal{K}$ and $f(s) \in H_{0}(K)$. Then the limit

$$
\begin{gathered}
\lim _{N \rightarrow \infty} \frac{1}{N+1} \#\left\{0 \leqslant k \leqslant N: \sup _{s \in K}|\zeta(s+i k h ; \mathfrak{a})-f(s)|<\varepsilon\right\} \\
=m_{H}\left\{\omega \in \Omega: \sup _{s \in K}|\zeta(s, \omega ; \mathfrak{a})-f(s)|<\varepsilon\right\}>0
\end{gathered}
$$

exists for all but at most countably many $\varepsilon>0$.

Proof of Theorem 2 easily follows from a discrete limit theorem (Theorem 3 of [4]) by using the equivalent of weak convergence of probability measures in terms of continuity sets.

\section{Approximation in the mean}

Theorem 1 will be derived from Theorem 2. For this, we need a certain approximation result of $\zeta_{u_{N}}(s ; \mathfrak{a})$ by $\zeta(s ; \mathfrak{a})$.

Lemma 1. Suppose that $u_{N} \rightarrow \infty, u_{N} \ll N^{2} \log ^{A} N$ with every $A>0$ as $N \rightarrow \infty$. Then, for every compact set $K \subset D$ and $h>0$,

$$
\lim _{N \rightarrow \infty} \frac{1}{N+1} \sum_{k=0}^{N} \sup _{s \in K}\left|\zeta(s+i k h ; \mathfrak{a})-\zeta_{u_{N}}(s+i k h ; \mathfrak{a})\right|=0 .
$$

Proof. Let $1 / 2<\sigma<1$ be fixed and $T \rightarrow \infty$. Then it is well known that

$$
\int_{-T}^{T}|\zeta(\sigma+i t, \alpha)|^{2} \mathrm{~d} t \ll_{\sigma, \alpha} T, \quad \int_{-T}^{T}\left|\zeta^{\prime}(\sigma+i t, \alpha)\right|^{2} \mathrm{~d} t \ll_{\sigma, \alpha} T .
$$

From these estimates and (1.1), we have

$$
\int_{-T}^{T}|\zeta(\sigma+i t ; \mathfrak{a})|^{2} \mathrm{~d} t \ll_{\sigma, \mathfrak{a}} T, \quad \int_{-T}^{T}\left|\zeta^{\prime}(\sigma+i t ; \mathfrak{a})\right|^{2} \mathrm{~d} t \ll_{\sigma, \mathfrak{a}} T .
$$


Hence, for $\tau \in \mathbb{R}$,

$$
\begin{aligned}
& \int_{0}^{T}|\zeta(\sigma+i \tau+i t ; \mathfrak{a})|^{2} \mathrm{~d} t \ll_{\sigma, \mathfrak{a}} T(1+|\tau|), \\
& \int_{0}^{T}\left|\zeta^{\prime}(\sigma+i \tau+i t ; \mathfrak{a})\right|^{2} \mathrm{~d} t \ll_{\sigma, \mathfrak{a}} T(1+|\tau|) .
\end{aligned}
$$

Now the latter estimates and the application of the Gallagher lemma that connects discrete and continuous mean squares for some differentiable functions (Lemma 1.4 of [8]), lead to the bound

$$
\begin{aligned}
& \sum_{k=0}^{N}|\zeta(\sigma+i k h+i \tau ; \mathfrak{a})|^{2} \ll_{h} \int_{0}^{N h}|\zeta(\sigma+i \tau+i t ; \mathfrak{a})|^{2} \mathrm{~d} t \\
& \quad+\left(\int_{0}^{N h}|\zeta(\sigma+i \tau+i t ; \mathfrak{a})|^{2} \mathrm{~d} t \int_{0}^{N h}\left|\zeta^{\prime}(\sigma+i \tau+i t ; \mathfrak{a})\right|^{2} \mathrm{~d} t\right)^{1 / 2} \\
& \quad \ll_{\sigma, \mathfrak{a}, h} N(1+|\tau|) .
\end{aligned}
$$

Let $K \subset D$ be an arbitrary compact set. Then there exists $\varepsilon>0$ such that $K$ lies in the strip $\{s \in \mathbb{C}: 1 / 2+2 \varepsilon \leqslant \sigma \leqslant 1-\varepsilon\}$. For $s$ from that strip, we have $\theta_{1}=\sigma-\frac{1}{2}-\varepsilon>0$. Therefore, the representation (2.2) and the residue theorem give

$$
\zeta_{u_{N}}(s ; \mathfrak{a})-\zeta(s ; \mathfrak{a})=\frac{1}{2 \pi i} \int_{-\theta_{1}-i \infty}^{-\theta_{1}+i \infty} \zeta(s+z ; \mathfrak{a}) l_{u_{N}}(z) \frac{\mathrm{d} z}{z}+\frac{\hat{a} l_{u_{N}}(1-s)}{1-s} .
$$

Therefore, for $s \in K$,

$$
\begin{aligned}
& \left|\zeta(s+i k h ; \mathfrak{a})-\zeta_{u_{N}}(s+i k h ; \mathfrak{a})\right| \\
& \ll \int_{-\infty}^{\infty}\left|\zeta\left(\frac{1}{2}+\varepsilon+i k h+i t+i \tau ; \mathfrak{a}\right)\right| \frac{\left|l_{u_{N}}(1 / 2+\varepsilon-\sigma+i \tau)\right|}{|1 / 2+\varepsilon-\sigma+i \tau|} \mathrm{d} \tau \\
& \quad+\frac{|\hat{a}|\left|l_{u_{N}}(1-s-i k h)\right|}{|1-s-i k h|} \\
& \ll \int_{-\infty}^{\infty}\left|\zeta\left(\frac{1}{2}+\varepsilon+i k h+i \tau ; \mathfrak{a}\right)\right| \sup _{s \in K} \frac{\left|l_{u_{N}}(1 / 2+\varepsilon-s+i \tau)\right|}{|1 / 2+\varepsilon-s+i \tau|} \mathrm{d} \tau \\
& \quad+\frac{|\hat{a}|\left|l_{u_{N}}(1-s-i k h)\right|}{|1-s-i k h|} .
\end{aligned}
$$

Hence,

$$
\begin{aligned}
& \frac{1}{N+1} \sum_{k=0}^{N}\left|\zeta(s+i k h ; \mathfrak{a})-\zeta_{u_{N}}(s+i k h ; \mathfrak{a})\right| \\
& \ll \int_{-\infty}^{\infty}\left(\frac{1}{N+1} \sum_{k=0}^{N}\left|\zeta\left(\frac{1}{2}+\varepsilon+i k h+i \tau ; \mathfrak{a}\right)\right|\right) \sup _{s \in K} \frac{\left|l_{u_{N}}(1 / 2+\varepsilon-s+i \tau)\right|}{|1 / 2+\varepsilon-s+i \tau|} \mathrm{d} \tau \\
& \quad+|\hat{a}| \frac{1}{N+1} \sum_{k=0}^{N} \sup _{s \in K} \frac{\left|l_{u_{N}}(1-s-i k h)\right|}{|1-s-i k h|} \stackrel{\text { def }}{=} I+Z
\end{aligned}
$$


The Cauchy inequality and (4.1) show that

$$
\begin{aligned}
& \frac{1}{N+1} \sum_{k=0}^{N}\left|\zeta\left(\frac{1}{2}+\varepsilon+i k h+i \tau ; \mathfrak{a}\right)\right| \\
& \leqslant\left(\frac{1}{N+1} \sum_{k=0}^{N}\left|\zeta\left(\frac{1}{2}+\varepsilon+i k h+i \tau ; \mathfrak{a}\right)\right|^{2}\right)^{1 / 2} \ll_{\varepsilon, \mathfrak{a}, h}(1+|\tau|)^{1 / 2} .
\end{aligned}
$$

It is well known that there exists a constant $c>0$ such that

$$
\Gamma(\sigma+i t) \ll \exp \{-c|t|\}
$$

uniformly in $\sigma_{1}<\sigma<\sigma_{2}$ for arbitrary $\sigma_{1}<\sigma_{2}$. Thus, by the definition of $l_{u}(s)$, for all $s \in K$,

$$
\begin{aligned}
\frac{l_{u_{N}}(1 / 2+\varepsilon-s+i \tau)}{1 / 2+\varepsilon-s+i \tau} & \ll{ }_{\theta} u_{N}^{1 / 2+\varepsilon-\sigma}\left|\Gamma\left(\frac{1}{\theta}\left(\frac{1}{2}+\varepsilon-i t+i \tau\right)\right)\right| \\
& \ll_{\theta} u_{N}^{-\varepsilon} \exp \left\{-\frac{c}{\theta}|\tau-t|\right\} \ll_{\theta, K} u_{N}^{-\varepsilon} \exp \left\{-\frac{c}{\theta}|\tau|\right\} .
\end{aligned}
$$

This together with (4.3) implies the bound

$$
I \ll_{\varepsilon, \mathfrak{a}, h, \theta, K} u_{N}^{-\varepsilon} \int_{-\infty}^{\infty}(1+|\tau|)^{1 / 2} \exp \left\{-\frac{c}{\theta}|\tau|\right\} \mathrm{d} \tau \ll_{\varepsilon, \mathfrak{a}, h, \theta, K} u_{N}^{-\varepsilon} .
$$

Similarly, using (4.4), we obtain that, for all $s \in K$,

$$
\frac{l_{u_{N}}(1-\sigma-i t+i k h)}{1-\sigma-i t+i k h} \ll_{\theta, K} u_{N}^{1-\sigma} \exp \left\{-\frac{c h}{\theta} k\right\} .
$$

Therefore,

$$
Z \ll_{\theta, K, \mathfrak{a}} \frac{u_{N}^{1 / 2-2 \varepsilon}}{N} \sum_{k=0}^{N} \exp \left\{-\frac{c h}{\theta} k\right\} \ll_{\theta, K, \mathfrak{a}, h} u_{N}^{1 / 2-2 \varepsilon} \frac{\log N}{N} .
$$

Since $u_{N} \ll N^{2} \log ^{A} N$, this together with (4.5) and (4.2) proves the lemma.

\section{Proof of universality}

For the proof of Theorem 1, we will apply the probabilistic approach based on weak convergence of probability measures and distribution functions. First we will recall the proof of Theorem 2 .

For $A \in \mathcal{B}(H(D))$, define

$$
P_{N, \mathfrak{a}, h}(A)=\frac{1}{N+1} \#\{0 \leqslant k \leqslant N: \zeta(s+i k h ; \mathfrak{a}) \in A\},
$$

and let $P_{\zeta, \mathfrak{a}}$ denote the distribution of the random element $\zeta(s, \omega ; \mathfrak{a})$, i. e.,

$$
P_{\zeta, \mathfrak{a}}(A)=m_{H}\{\omega \in \Omega: \zeta(s, \omega ; \mathfrak{a}) \in A\} .
$$


Lemma 2. Suppose that the sequence $\mathfrak{a}$ is multiplicative and the set $L(\mathbb{P}, h, \pi)$ is linearly independent over $\mathbb{Q}$. Then $P_{N, \mathfrak{a}, h}$ converges weakly to $P_{\zeta, \mathfrak{a}}$ as $N \rightarrow$ $\infty$. Moreover, the support of the measure $P_{\zeta, \mathfrak{a}}$ is the set $\{g \in H(D): g(s) \neq$ 0 or $g(s) \equiv 0\} \stackrel{\text { def }}{=} S$.

Proof. The first assertion of the lemma is contained in Theorem 3 of [4] with weight function $w(u) \equiv 1$. Observe that in [4] the restriction $h \geqslant 1$ in the definition of the set $L(\mathbb{P}, h, \pi)$ is involved, however, in the case $w(u) \equiv 1$, the latter restriction is not needed.

The second assertion of the lemma can be found in [2].

Proof. (Proof of Theorem 2). Since $f(s) \neq 0$ on $K$, by the Mergelyan theorem on the approximation of analytic functions by polynomials [7], there exists a polynomial $p(s)$ such that

$$
\sup _{s \in K}\left|f(s)-\mathrm{e}^{p(s)}\right|<\frac{\varepsilon}{2} .
$$

Obviously, $\mathrm{e}^{p(s)} \in S$. Therefore, the set

$$
G_{\varepsilon}=\left\{g \in H(D): \sup _{s \in K}\left|g(s)-\mathrm{e}^{p(s)}\right|<\frac{\varepsilon}{2}\right\}
$$

is open and contains an element of the support of the measure $P_{\zeta, \mathfrak{a}}$. Hence,

$$
P_{\zeta, \mathfrak{a}}\left(G_{\varepsilon}\right)>0 .
$$

Define the open neighbourhood of the function $f(s)$

$$
\mathcal{G}_{\varepsilon}=\left\{g \in H(D): \sup _{s \in K}|g(s)-f(s)|<\varepsilon\right\} .
$$

The boundary $\partial \mathcal{G}_{\varepsilon}$ of the set $\mathcal{G}_{\varepsilon}$ is the set

$$
\left\{g \in H(D): \sup _{s \in K}|g(s)-f(s)|=\varepsilon\right\} .
$$

Therefore, the boundaries $\partial \mathcal{G}_{\varepsilon_{1}}$ and $\partial \mathcal{G}_{\varepsilon_{2}}$ do not intersect for different positive $\varepsilon_{1}$ and $\varepsilon_{2}$. This implies that $P_{\zeta, \mathfrak{a}}\left(\partial \mathcal{G}_{\varepsilon}\right)=0$ for all but at most countably many $\varepsilon>0$, i. e., in other words, the set $\mathcal{G}_{\varepsilon}$ is a continuity set of the measure $P_{\zeta, \mathfrak{a}}$ for all but at most countably many $\varepsilon>0$. Thus, an application of the first assertion of Lemma 2 together with the equivalent of weak convergence of probability measures in terms of continuity sets, see, for example, [1], gives

$$
\lim _{N \rightarrow \infty} P_{N, \mathfrak{a}, h}\left(\mathcal{G}_{\varepsilon}\right)=P_{\zeta, \mathfrak{a}}\left(\mathcal{G}_{\varepsilon}\right)
$$

for all but at most countably many $\varepsilon>0$. Moreover, in view of (5.1),

$$
\sup _{s \in K}|g(s)-f(s)| \leqslant \sup _{s \in K}\left|g(s)-\mathrm{e}^{p(s)}\right|+\sup _{s \in K}\left|f(s)-\mathrm{e}^{p(s)}\right|<\varepsilon
$$


for $g \in \mathcal{G}_{\varepsilon}$. This shows that $\mathcal{G}_{\varepsilon} \supset G_{\varepsilon}$. Therefore, by (5.2), we have the inequality $P_{\zeta, \mathfrak{a}}\left(\mathcal{G}_{\varepsilon}\right)>0$. This inequality, the definitions of $P_{N, \mathfrak{a}, h}$ and $\mathcal{G}_{\varepsilon}$, and (5.3) prove the theorem.

We observe that the limit measure $P_{\zeta, \mathfrak{a}}$ is independent of the number $h$, and this is conditioned by the linear independence over $\mathbb{Q}$ of the set $L(\mathbb{P}, h, \pi)$. The case of the linear dependence of the set $L(\mathbb{P}, h, \pi)$ is more complicated and requires a new probability space in place of $\left(\Omega, \mathcal{B}(\Omega), m_{H}\right)$. This case will be considered elsewhere.

Proof. (Proof of Theorem 1). We will deduce Theorem 1 from Theorem 2 by using Lemma 1 and the weak convergence of distribution functions.

Define the functions

$$
\begin{aligned}
F_{N, \mathfrak{a}, h}(\varepsilon) & =P_{N, \mathfrak{a}, h}\left(\mathcal{G}_{\varepsilon}\right) \\
& =\frac{1}{N+1} \#\left\{0 \leqslant k \leqslant N: \sup _{s \in K}|\zeta(s+i k h ; \mathfrak{a})-f(s)|<\varepsilon\right\}, \\
\hat{F}_{N, \mathfrak{a}, h}(\varepsilon) & =\frac{1}{N+1} \#\left\{0 \leqslant k \leqslant N: \sup _{s \in K}\left|\zeta_{u_{N}}(s+i k h ; \mathfrak{a})-f(s)\right|<\varepsilon\right\}, \\
F_{\zeta, \mathfrak{a}}(\varepsilon) & =P_{\zeta, \mathfrak{a}}\left(\mathcal{G}_{\varepsilon}\right)=m_{H}\left\{\omega \in \Omega: \sup _{s \in K}|\zeta(s, \omega ; \mathfrak{a})-f(s)|<\varepsilon\right\} .
\end{aligned}
$$

The functions $F_{N, \mathfrak{a}, h}(\varepsilon), \hat{F}_{N, \mathfrak{a}, h}(\varepsilon)$ and $F_{\zeta, \mathfrak{a}}(\varepsilon)$ as functions of the variable $\varepsilon$ are distribution functions. Actually, they are non-decreasing, left continuous, at $+\infty$ take values 1 and at $-\infty$ take values 0 . Moreover, since

$$
\begin{aligned}
& \partial \mathcal{G}_{\varepsilon} \\
& =\left\{g \in H(D): \sup _{s \in K}|g(s)-f(s)| \leqslant \varepsilon\right\} \backslash\left\{g \in H(D): \sup _{s \in K}|g(s)-f(s)|<\varepsilon\right\},
\end{aligned}
$$

we have

$$
\begin{aligned}
& P_{\zeta, \mathfrak{a}}\left(\partial \mathcal{G}_{\varepsilon}\right)=m_{H}\left\{\omega \in \Omega: \sup _{s \in K}|\zeta(s, \omega ; \mathfrak{a})-f(s)| \leqslant \varepsilon\right\} \\
& -m_{H}\left\{\omega \in \Omega: \sup _{s \in K}|\zeta(s, \omega ; \mathfrak{a})-f(s)|<\varepsilon\right\}=F_{\zeta, \mathfrak{a}}(\varepsilon+0)-F_{\zeta, \mathfrak{a}}(\varepsilon) .
\end{aligned}
$$

Hence, $F_{\zeta, \mathfrak{a}}(\varepsilon+0)=F_{\zeta, \mathfrak{a}}(\varepsilon)$ if and only if $P_{\zeta, \mathfrak{a}}\left(\partial \mathcal{G}_{\varepsilon}\right)=0$. Therefore, the point $\varepsilon$ is a continuity point of the distribution function $F_{\zeta, \mathfrak{a}}$ if and only if the set $\mathcal{G}_{\varepsilon}$ is a continuity set of the measure $P_{\zeta, \mathfrak{a}}$. By $(5.3), P_{N, \mathfrak{a}, h}$ converges weakly to $P_{\zeta, \mathfrak{a}}$ as $N \rightarrow \infty$ for all continuity sets $\mathcal{G}_{\varepsilon}$ of $P_{\zeta, \mathfrak{a}}$. Thus, by the above remarks, the distribution function $F_{N, \mathfrak{a}, h}(\varepsilon)$ as $N \rightarrow \infty$ converges to the distribution function $F_{\zeta, \mathfrak{a}}(\varepsilon)$ at all its continuity points $\varepsilon\left(F_{N, \mathfrak{a}, h}(\varepsilon)\right.$ converges weakly to $F_{\zeta, \mathfrak{a}}(\varepsilon)$ as $\left.N \rightarrow \infty\right)$.

Denote by $\varphi_{N, \mathfrak{a}, h}(v), \hat{\varphi}_{N, \mathfrak{a}, h}(v)$ and $\varphi_{\zeta, \mathfrak{a}}(v), v \in \mathbb{R}$, the characteristic functions of the distribution functions $F_{N, \mathfrak{a}, h}(\varepsilon), \hat{F}_{N, \mathfrak{a}, h}(\varepsilon)$ and $F_{\zeta, \mathfrak{a}}(\varepsilon)$, respectively. Since $F_{N, \mathfrak{a}, h}(\varepsilon)$ converges weakly to $F_{\zeta, \mathfrak{a}}(\varepsilon)$ as $N \rightarrow \infty$, the continuity theorem for characteristic functions implies the equality

$$
\lim _{N \rightarrow \infty} \varphi_{N, \mathfrak{a}, h}(v)=\varphi_{\zeta, \mathfrak{a}}(v)
$$


for all $v \in \mathbb{R}$. We have to show that $\hat{\varphi}_{N, \mathfrak{a}, h}(v)$ also converges to $\varphi_{\zeta, \mathfrak{a}}(v)$ as $N \rightarrow \infty$.

By the definition of characteristic functions,

$$
\begin{aligned}
\hat{\varphi}_{N, \mathfrak{a}, h}(v)-\varphi_{N, \mathfrak{a}, h}(v)= & \int_{-\infty}^{\infty} \mathrm{e}^{i v \varepsilon} \mathrm{d}\left(\hat{F}_{N, \mathfrak{a}, h}(\varepsilon)-F_{N, \mathfrak{a}, h}(\varepsilon)\right) \\
= & \frac{1}{N+1} \sum_{k=0}^{\infty}\left(\exp \left\{i v \sup _{s \in K}\left|\zeta_{u_{N}}(s+i k h ; \mathfrak{a})-f(s)\right|\right\}\right. \\
& \left.-\exp \left\{i v \sup _{s \in K}|\zeta(s+i k h ; \mathfrak{a})-f(s)|\right\}\right) .
\end{aligned}
$$

Hence,

$$
\begin{aligned}
& \left|\hat{\varphi}_{N, \mathfrak{a}, h}(v)-\varphi_{N, \mathfrak{a}, h}(v)\right| \leqslant \frac{1}{N+1} \sum_{k=0}^{N} \mid \exp \left\{i v \left(\sup _{s \in K}\left|\zeta_{u_{N}}(s+i k h ; \mathfrak{a})-f(s)\right|\right.\right. \\
& \left.\left.-\sup _{s \in K}|\zeta(s+i k h ; \mathfrak{a})-f(s)|\right)\right\}-1\left|\leqslant \frac{|v|}{N+1} \sum_{k=0}^{N}\right| \sup _{s \in K}\left|\zeta_{u_{N}}(s+i k h ; \mathfrak{a})-f(s)\right| \\
& -\sup _{s \in K}|\zeta(s+i k h ; \mathfrak{a})-f(s)|\left|\leqslant \frac{|v|}{N+1} \sum_{k=0}^{N} \sup _{s \in K}\right| \zeta_{u_{N}}(s+i k h ; \mathfrak{a})-\zeta(s+i k h ; \mathfrak{a}) \mid .
\end{aligned}
$$

Therefore, in view of Lemma 1,

$$
\hat{\varphi}_{N, \mathfrak{a}, h}(v)-\varphi_{N, \mathfrak{a}, h}(v)=o(1)
$$

as $N \rightarrow \infty$ uniformly in $|v| \leqslant C$ with every $\infty>C>0$. This and (5.4) show that

$$
\lim _{N \rightarrow \infty} \hat{\varphi}_{N, \mathfrak{a}, h}(v)=\varphi_{\zeta, \mathfrak{a}}(v)
$$

uniformly in $|v| \leqslant C$. Therefore, by the continuity theorem, we obtain that the distribution function $\hat{F}_{N, \mathfrak{a}, h}(\varepsilon)$ converges weakly to $F_{\zeta, \mathfrak{a}}(\varepsilon)$ as $N \rightarrow \infty$, or $\hat{F}_{N, \mathfrak{a}, h}(\varepsilon)$ converges weakly to $F_{\zeta, \mathfrak{a}}(\varepsilon)$ in all continuity points $\varepsilon$ of $F_{\zeta, \mathfrak{a}}(\varepsilon)$. However, the distribution function has at most countably many of discontinuity points. This and the definitions of $\hat{F}_{N, \mathfrak{a}, h}(\varepsilon)$ and $F_{\zeta, \mathfrak{a}}(\varepsilon)$ prove the theorem.

\section{Acknowledgements}

The research of the second author is funded by the European Social Fund (project No. 09.3.3-LMT-K-712-01-0037) under grant agreement with the Research Council of Lithuania (LMT LT).

\section{References}

[1] P. Billingsley. Convergence of Probability Measures. Willey, New York, 1968.

[2] A. Laurinčikas. Limit Theorems for the Riemann Zeta-Function. Kluwer, Dordrecht, 1996. https://doi.org/10.1007/978-94-017-2091-5. 
[3] A. Laurinčikas. The joint discrete universality of periodic zeta-functions. In J. Sander, J. Steuding and R. Steuding(Eds.), From Arithmetic to Zeta-Functions, Number Theory in Memory of Wolfgang Schwarz, pp. 231-246. Springer, 2016. https://doi.org/10.1007/978-3-319-28203-9_15.

[4] R. Macaitienè, M. Stoncelis and D. Šiaučiūnas. A weighted discrete universality theorem for periodic zeta-functions. In A. Dubickas, A. Laurinčikas, E. Manstavičius and G. Stepanauskas(Eds.), Anal. Probab. Methods Number Theory, Proc. 6th Int. Conf., Palanga, Lithuania, 11-17 September 2016, pp. 97-107, Vilnius, 2017. VU Leidykla.

[5] R. Macaitienè, M. Stoncelis and D. Šiaučiūnas. A weighted discrete universality theorem for periodic zeta-functions. II. Mathematical Modelling and Analysis, 22(6):750-762, 2017. https://doi.org/10.3846/13926292.2017.1365779.

[6] K. Matsumoto. A survey on the theory of universality for zeta and $L$-functions. In M. Kaneko, S. Kanemitsu and J. Liu(Eds.), Number Theory: Plowing and Starring Through High Wawe Forms, Proc. Tth China-Japan Semin. (Fukuoka 2013), volume 11 of Number Theory and its Applications, pp. 95-144, New Jersey, London, Singapore, Beijing, Shanghai, Hong Kong, Taipei, Chennai, 2015. World Scientific Publishing Co. https://doi.org/10.1142/9789814644938_0004.

[7] S.N. Mergelyan. Uniform approximations to functions of complex variable. Usp. Mat. Nauk., 7(2):31-122, 1952 (in Russian).

[8] H.L. Montgomery. Topics in Multiplicative Number Theory. Lecture Notes Math. Vol. 227, Springer-Verlag, Berlin, 1971. https://doi.org/10.1007/BFb0060851. 